\title{
CÁLCULO DO ÍNDICE OVERALL EQUIPMENT EFFETIVENESS (OEE) NA PRODUÇÃO DE UMA MÁQUINA DO SETOR TÊXTIL
}

\section{CALCULATION OF THE OVERALL EQUIPMENT EFFETIVENESS (OEE) INDEX ON THE PRODUCTION OF A TEXTILE SECTOR MACHINE}

Thiago Teixeira Lopes- thiagotlopes2016@gmail.com Faculdade de Tecnologia de Taquaritinga - Taquaritinga - São Paulo - Brasil

Carlos Roberto Regattieri - carlos.regattieri@fatec.sp.gov.br Faculdade de Tecnologia de Taquaritinga - Taquaritinga - São Paulo - Brasil

DOI: 10.31510/infa.v18i1.1103

Data de submissão: 14/04/2021

Data do aceite: 09/07/2021

Data da publicação: 30/07/2021

\section{RESUMO}

Este artigo apresenta a implementação da ferramenta de controle de paradas de máquina e posteriormente o cálculo do Indicador OEE - Overall Equipment Effetiveness, aplicado em uma máquina no setor de produção de telas agrícolas de sombreamento de uma empresa têxtil, localizada no distrito industrial III na cidade de Itápolis-SP. No mercado competitivo que vivemos, é muito importante conhecermos totalmente a capacidade produtiva da empresa. Com a implementação do Indicador OEE - Overall Equipment Effetiveness, podemos descobrir a "fábrica oculta", com isso podemos aumentar a produtividade e diminuir as perdas do processo produtivo, deixando assim a empresa mais competitiva no mercado. São demonstrados os índices de Disponibilidade, Desempenho e Qualidade, fazendo um comparativo entre o OEE encontrado e o OEE de uma Empresa Manufatura de Classe Mundial. Foi desenvolvido junto ao grupo de pesquisa em OEE montado pela empresa, a coleta de dados no chão de fábrica e análise de acordo com os parâmetros de OEE.

Palavras-chave: OEE. Disponibilidade. Desempenho. Qualidade. Produção.

\begin{abstract}
This article presents the implementation of the machine stops control tool and later the calculation of the OEE Indicator - Overall Equipment Effetiveness, also the elaboration of the A3 report, applied to a machine in the production sector of agricultural shading fabrics of a textile company, located in industrial district III in the city of Itápolis-SP. In the competitive market that we live in, it is very important to fully understand the company's productive capacity. With the implementation of the OEE Indicator - Overall Equipment Effetiveness, we can discover the "hidden factory", with this we can increase productivity and decrease losses in the production process, thus making the company more competitive in the market. The
\end{abstract}


Availability, Performance and Quality indices are demonstrated, making a comparison between the OEE found and the OEE of a World Class Manufacturing Company. It was developed together with the OEE research group set up by the company, the data collection on the shop floor and analysis according to the OEE parameters.

Keywords: OEE. Availability. Performance. Quality. Production.

\section{INTRODUÇÃO}

Diante do mercado competitivo que as empresas vivem, uma gestão estratégica na tomada de decisões, deixa a empresa sempre à frente dos seus concorrentes. Uma gestão bem aplicada, tanto de custo como estratégica em chão de fábrica, pode ser feita através de um indicador.

Este trabalho demonstrará o uso do Indicador OEE - Overall Equipment Effetiveness, em uma máquina Tear Raschel Liba Racop 4 no seu processo produtivo, a diferença entre o tempo disponível de produção e o tempo efetivo para produzir, considerando o tempo de desperdício. Abaixo na Figura 1, foto da Máquina que será utilizada neste processo.

Figura 01- Tear Raschel Liba Racop 4

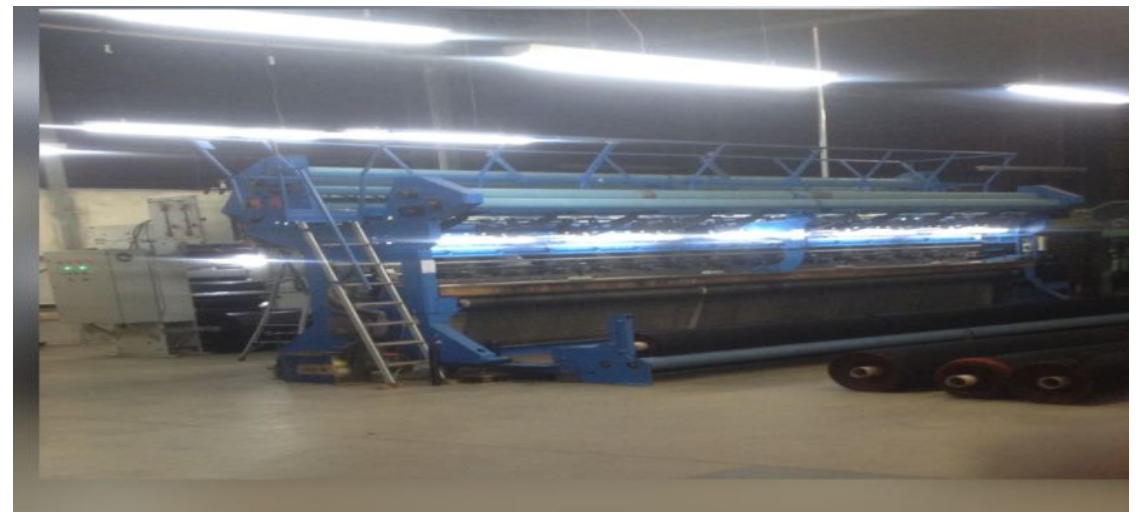

Fonte: Autor (2021)

O OEE tem três componentes segundo SILVA (2013), que são:

- Tempo útil que o equipamento tem para funcionar, ou seja, produzir que é a disponibilidade do equipamento;

- A eficiência durante o funcionamento, desempenho do equipamento;

- A qualidade do produto resultado do processo; 
De acordo com Amorim (2009) o OEE é um indicador que faz a medição do desempenho de uma maneira "tridimensional" uma vez que considera quanto tempo útil a máquina tem para produzir, a eficiência que demonstra durante a produção e a qualidade que é obtida no produto após o procedimento do equipamento.

A metodologia OEE tem como objetivo principal, encontrar o problema raiz de cada situação de perda do processo e posteriormente definir uma ação corretiva eficaz podendo essa ser feita através do relatório A3.

A Figura abaixo demonstra o indicador OEE, bem como seus índices e as perdas que se relacionam com cada um deles.

Figura 2: Elementos da Eficácia Global de uma Máquina

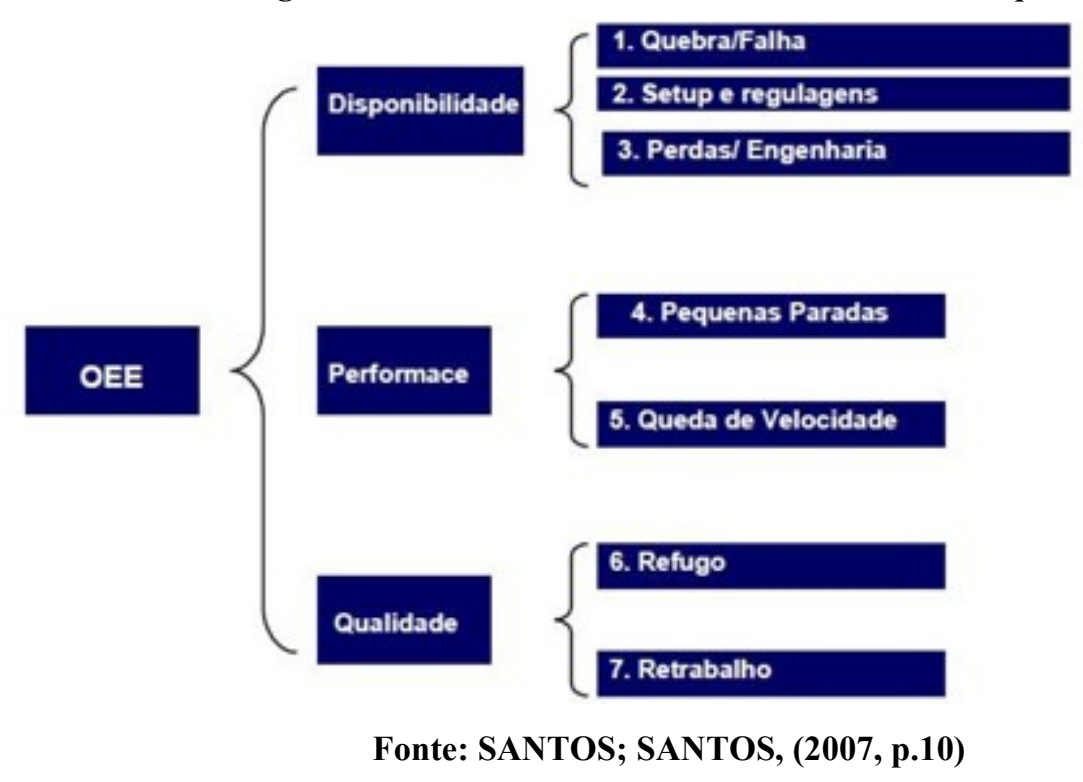

\section{COLETA DE DADOS}

O trabalho foi feito através da coleta de dados na referida empresa, pelo grupo de pesquisa em OEE montado pela mesma, analisando o equipamento em questão e observando o processo produtivo. Com a elaboração de planilhas com o software Microsoft Excel, foi 
possível trabalhar os dados coletados e posteriormente aplicá-los nos parâmetros do indicador OEE.

A pesquisa científica, de acordo com Ruiz (1991): "É a realização concreta de uma investigação planejada, desenvolvida e redigida de acordo com as normas da metodologia consagradas pela ciência".

A coleta de dados foi baseada em:

1 - Identificação de todas as perdas do processo, previstas e não previstas;

2- Cronometragem de todo o processo produtivo, identificando e apontando em planilha cada situação;

3- Cálculos segundo o conceito estabelecido através do estudo da arte.

Segundo Nakajima (1989) é possível relacionar seis grandes perdas nos processos de produção e seus impactos em cada componente do Indicador OEE, como apresenta a Figura 3:

Figura 3: Relação entre as seis grandes perdas e os fatores do OEE.
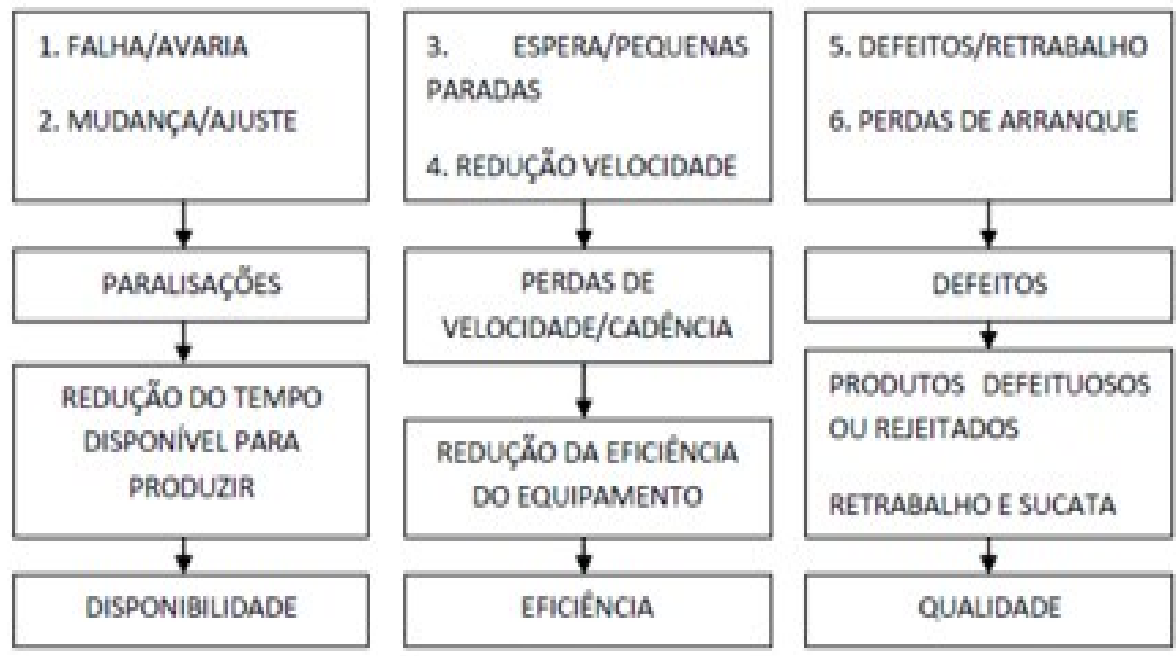

Fonte: Adaptado de Amorim (2009)

\section{CÁLCULO DO ÍNDICE DE OEE}

Para este trabalho foram realizadas medidas na máquina denominado tear Raschel Liba Racop 4, em uma Indústria Têxtil no período de Janeiro/2021. 


\subsection{Cálculo da Disponibilidade, Desempenho e Qualidade}

A empresa no qual foi realizado o trabalho, trabalha no sistema de produção puxada, tendo um período disponível de produção de 24 horas por dia, pois a mesma trabalha com sistema de revezamento de operador de máquina nos horários de refeição. Sendo assim, foi fixado para o cálculo do OEE, o Desempenho em 95\%, tendo em vista que são máquinas automáticas e a Qualidade em 99\%, ficando apenas a medida de disponibilidade a ser mensurada, sendo assim, considerado as paradas programadas e não programadas.

Santos e Santos (2007) considera que o índice ideal para o indicador OEE deve ser de 85\% (Classe Mundial), e que para alcançar esse índice os valores dos fatores para o cálculo sejam, por exemplo: disponibilidade $=90 \%$, eficiência $=95 \%$ e qualidade $=99 \%$.

\subsection{Cálculo das paradas programadas e paradas não programadas}

Paradas Programadas são aquelas que já foram previstas e calculadas na gestão tanto de custo como estratégica no chão de fábrica, porém devem ser medidas e calculadas para que se possa melhorar ou otimizar a mesma. Paradas não programadas são aquelas que não foram previstas e nem calculadas pela gestão de custo e estratégica no chão de fábrica, essas são consideradas como perdas do processo. Podemos citar como exemplo de perdas programadas, o intervalo para alimentação do operador e manutenção programada do equipamento, já para perdas não programadas, podemos citar como exemplo, parada por problema técnico no equipamento, ociosidades (aguardando operador), sem recursos (falta de energia elétrica) entre outras.

Um modelo de como foram registradas essas paradas são as Figuras 4, 5 e 6. 
Figura 4 - Tabela de Cronometragem do tempo de paradas

\begin{tabular}{|c|c|c|}
\hline $\begin{array}{c}\text { Duração } \\
\text { minutos }\end{array}$ & Tipo de apontamento / Motivo & Observação \\
\hline 00:10:45 & Parada-Troca de bobina & Solda bobina 8 \\
\hline 00:00:41 & Parada-Quebra de fio no tear & Solda estourou \\
\hline 00:00:32 & Parada-Quebra de fio no iso & Solda \\
\hline 00:03:04 & Parada-Quebra de fio no tear & Solda \\
\hline 00:01:14 & Parada-Quebra de fio no iso & Solda \\
\hline 00:01:06 & Parada-Quebra de fio no tear & Solda \\
\hline 00:01:39 & Parada-Quebra de fio no tear & Solda \\
\hline 00:05:03 & Parada-Quebra de fio no tear & Fio estourou \\
\hline $00: 01: 12$ & Parada-Quebra de fio no tear & Fio estourou \\
\hline $00: 02: 59$ & Parada-Quebra de fio no tear & Fio estourou \\
\hline $00: 00: 48$ & Parada-Quebra de fio no tear & Fio estourou \\
\hline $00: 01: 21$ & Parada-Quebra de fio no tear & Fio estourou \\
\hline $00: 01: 04$ & Parada-Quebra de fio no tear & Fio estourou \\
\hline $00: 01: 27$ & Parada-Quebra de fio no tear & \\
\hline
\end{tabular}

Fonte: Autor (2021)

Figura 5 - Tabela de paradas programadas

\begin{tabular}{|cc|}
\hline \multicolumn{2}{|c|}{ PARADA PROGRAMADA } \\
04:10:00 & 250 \\
Parada-Setup \\
Parada-Troca de bobina \\
00:41:07 \\
Parada-Troca do jumbo \\
17,42 \\
00:17:25 \\
\hline \multicolumn{2}{|c|}{$05: 08: 32$} \\
\hline
\end{tabular}

Fonte: Autor (2021)

Figura 6 - Tabela de paradas não programadas

\begin{tabular}{|c|c|}
\hline \multicolumn{2}{|c|}{ PARADA NÃO PROGRAMADA } \\
\hline \multicolumn{2}{|c|}{ Parada-Quebra de fio no tear } \\
\hline $04: 42: 59$ & 282,98 \\
\hline \multicolumn{2}{|c|}{ Parada-Quebra de fio no iso } \\
\hline $02: 38: 57$ & 158,95 \\
\hline \multicolumn{2}{|c|}{ Parada-Aguardando operador } \\
\hline $00: 48: 27$ & 48,45 \\
\hline Total & $8: 10: 23$ \\
\hline
\end{tabular}

Fonte: Autor (2021)

Interface Tecnológica - v. 18 n. 1 (2021) 
A Figura 4 mostra a lista de cronometragem de todas as paradas do equipamento, a Figura 5 mostra as paradas programadas, a Figura 6 as paradas não programadas, que contém o tempo total de cada uma delas dentro de todos os dias medidos e na figura 7 o resumo do tempo total do equipamento parado.

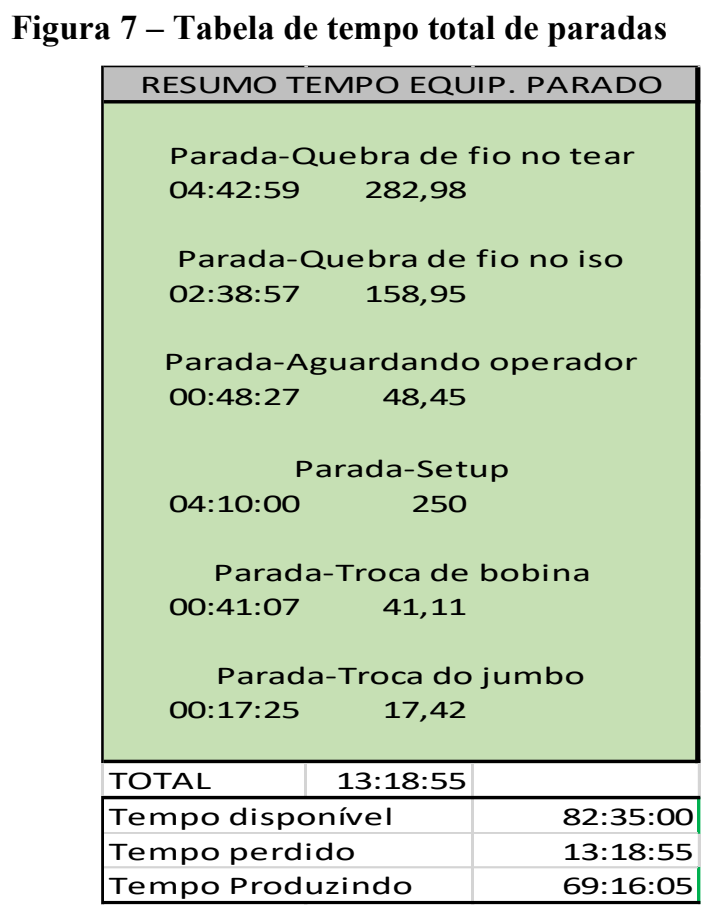

Fonte: Autor (2021)

\subsection{Cálculo do OEE}

A Figura 8 mostra a lista de cronometragem de todas as paradas e tempo de produção do equipamento. 


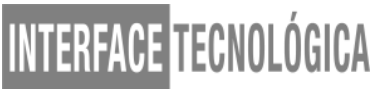

Figura 8 - Tabela de apontamento de tempo para cálculo do OEE

\begin{tabular}{|c|c|c|c|c|c|c|}
\hline \multirow[b]{2}{*}{ Evento \# } & \multirow{2}{*}{$\begin{array}{l}\text { Duração } \\
\text { Minutos }\end{array}$} & \multicolumn{2}{|c|}{ Quantidade } & \multirow[b]{2}{*}{ Produto } & \multirow[b]{2}{*}{ Tipo de Apontamento / motivo } & \multirow[b]{2}{*}{ Taxa } \\
\hline & & Boas & Ruins & & & \\
\hline 1 & 4156,08 & 2555,83 & 0 & $A$ & Produzindo & $83,88 \%$ \\
\hline 2 & 250 & 0 & 0 & $A$ & Parada-Setup & $5,05 \%$ \\
\hline 3 & 158,95 & 0 & 0 & $A$ & Parada-Quebra de fio no ISO & $3,21 \%$ \\
\hline 4 & 282,98 & 0 & 0 & A & Parada-Quebra de fio no tear & $5,71 \%$ \\
\hline 5 & 41,11 & 0 & 0 & $A$ & Parada-Troca de bobina & $0,83 \%$ \\
\hline 6 & 0 & 0 & 0 & $A$ & Parada-Manutenção & $0,00 \%$ \\
\hline 7 & 0 & 0 & 0 & A & Parada-Falta de energia & $0,00 \%$ \\
\hline 8 & 0 & 0 & 0 & $A$ & Parada-Check list & $0,00 \%$ \\
\hline 9 & 17,42 & 0 & 0 & $A$ & Parada-Troca de jumbo & $0,35 \%$ \\
\hline 10 & 0 & 0 & 0 & $A$ & Parada-Aguardando manutenção & $0,00 \%$ \\
\hline 11 & 0 & 0 & 0 & $A$ & Parada-Falta de MP & $0,00 \%$ \\
\hline 12 & 48,45 & 0 & 0 & $A$ & Parada-Aguardando operador & $0,98 \%$ \\
\hline 13 & 0,01 & 0 & 0 & Nenhum & Parada-Horários não apontados & \\
\hline
\end{tabular}

Fonte: Autor (2021)

A Figura 9 mostra a lista de resultado dos indicadores e também o resultado do OEE do equipamento.

Figura 9 - Tabela de indicadores do OEE

\begin{tabular}{|l|c|}
\hline 3. Cálculo OEE - Indicadores \\
\hline Utilização \%: & \\
\hline Disponibilidade \%: & $100,00 \%$ \\
\hline Performance\% & $83,88 \%$ \\
\hline Qualidade \%: & $91,63 \%$ \\
\hline OEE \%: & $100,00 \%$ \\
\hline
\end{tabular}

Fonte: Autor (2021)

A Figura 10 mostra o gráfico de desempenho do OEE. 
Figura 10 - Gráfico de desempenho do OEE

\section{DESEMPENHO DE OEE: D\% $\times$ P\% $\times$ Q\%}

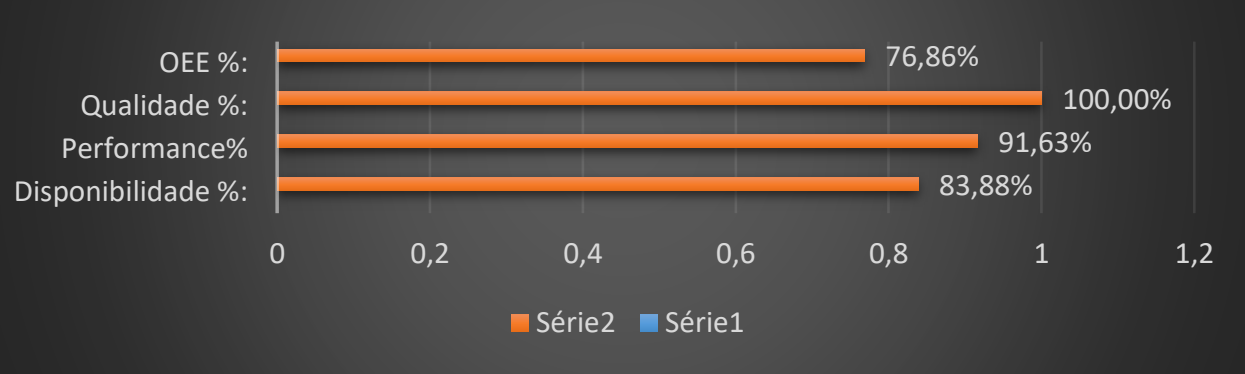

Fonte: Autor (2021)

A Figura 11 mostra o comparativo entre o OEE do equipamento e a tendência mundial.

Figura 11 - Tabela de comparação do OEE do equipamento e a tendência mundial

\begin{tabular}{|c|c|c|}
\hline Equipamento & OEE do Equipamento & Tendência mundial \\
\hline Tear Liba Racop 4 & $76,86 \%$ & $85,00 \%$ \\
\hline
\end{tabular}

Fonte: Autor (2021)

\section{RESULTADOS E ANÁLISE}

A partir dos dados coletados dos tempos de paradas da máquina Tear Raschel Liba Racop 4, a Figura 12 demonstra o índice em Percentual de cada tipo de parada sobre o tempo total do equipamento parado.

. Figura 12 - Tabela de percentual de paradas

\begin{tabular}{|c|c|}
\hline Tipo de Apontamento & índice \\
\hline Parada-Setup & $5,05 \%$ \\
\hline Parada-Quebra de fio no ISO & $3,21 \%$ \\
\hline Parada-Quebra de fio no tear & $5,71 \%$ \\
\hline Parada-Troca de bobina & $0,83 \%$ \\
\hline Parada-Manutenção & $0,00 \%$ \\
\hline Parada-Falta de energia & $0,00 \%$ \\
\hline Parada-Check list & $0,00 \%$ \\
\hline Parada-Troca de jumbo & $0,35 \%$ \\
\hline Parada-Aguardando manutenção & $0,00 \%$ \\
\hline Parada-Falta de MP & $0,00 \%$ \\
\hline Parada-Aguardando operador & $0,98 \%$ \\
\hline
\end{tabular}

Fonte: Autor (2021) 
Foi elaborado um gráfico com os motivos das paradas do equipamento segundo o percentual de cada um, conforme mostra a Figura 13, para que todos os envolvidos no projeto de OEE possam enxergar com clareza, onde e como terão que ser feitas as melhorias no processo produtivo em estudo.

Figura 13 - Gráfico de índice percentual paradas

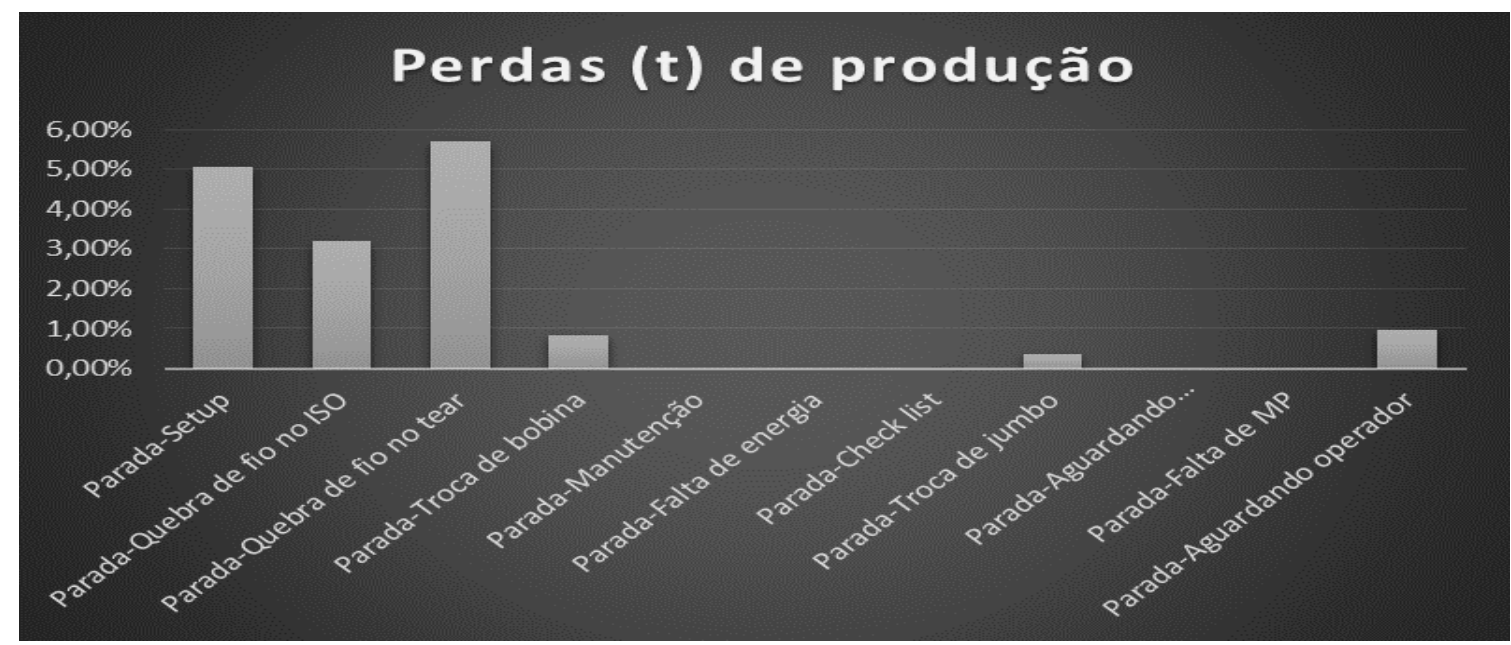

Fonte: Autor (2021)

Após os resultados das perdas do processo gerados pelo OEE, sugerido a implementação da melhoria e tratamento das perdas apontadas pelo indicador OEE através da ferramenta de melhoria Project A3.

É muito importante que seja coletado dados acerca de todo o tempo disponível do equipamento, para que assim seja coletados todas as paradas planejadas e não planejadas, a quantidade de produtos fabricados aprovados e não aprovados, podendo assim gerar informações de disponibilidade, performance e qualidade.

\section{CONCLUSÃO}

Esse estudo de caso mostrou através do OEE, que o equipamento no qual o estudo foi realizado, tem uma eficácia de 76,86\%. Esse equipamento era considerado na empresa, antes da implantação do OEE, a máquina que mais produzia e que menos tempo de produção perdido tinha, mas com o OEE foi possível identificar várias perdas ocultas que raramente eram apontadas. 
Conclui-se que é fundamental o uso da metodologia OEE em toda a empresa, visto que por meio de dados quantitativos pode-se ver se o uso do equipamento está sendo feito em sua total plenitude. O OEE mostrou a "fábrica oculta" que existe na empresa.

\section{REFERÊNCIAS}

AMORIM, J.P. OEE - A Forma de Medir a Eficiência dos Equipamentos. Disponível em: $<$ https://pt.scribd.com/doc/15122575/OEE-A-FORMA-DE-MEDIR-A-EFICACIA-DOSEQUIPAMENTOS $>$. Acesso em: 18/02/2021.

NAKAJIMA, Seiichi. Introdução ao TPM - Total Productive Maintenance. São Paulo: IMC Internacional Sistemas Educativos Ltda., 1989.

RUIZ, João Álvaro. Metodologia científica: guia para eficiência de estudos. 3 ed. São Paulo: Atlas, 1991.

SANTOS, A. C. O.; SANTOS, M. J. Utilização do Indicador de Eficácia Global de Equipamentos (OEE) na Gestão de Melhoria Contínua do Sistema de Manufatura. 2007. $10 \mathrm{f}$. UNIFEI.

SILVA, J.P.A.R. OEE -A forma de medir a eficácia dos equipamentos. Disponível em: $<$ http://pt.scribd.com/doc/15122575/OEE-A-FORMA-DE-MEDIR-A-EFICACIA-DOSEQUIPAMENTOS>. Acesso em: 18 set. 2018. 